\title{
A Model Derived from Hydrodynamic Simulations for Extracting the Size of Spherical Particles from the Quartz Crystal Microbalance
}

\author{
Jurriaan J.J. Gillissen ${ }^{1,2,4}$, Seyed R. Tabaei ${ }^{1,2}$, Joshua A. Jackman ${ }^{1,2}$, Nam-Joon Cho ${ }^{* 1,2,3}$
}

${ }^{1}$ School of Materials Science and Engineering, Nanyang Technological University, 50 Nanyang Avenue 639798, Singapore

${ }^{2}$ Centre for Biomimetic Sensor Science, Nanyang Technological University, 50 Nanyang Drive 637553, Singapore

${ }^{3}$ School of Chemical and Biomedical Engineering, Nanyang Technological University, 62 Nanyang Drive 637459, Singapore

Present Address:

${ }^{4}$ Center for Environmental Sensing and Modeling (CENSAM) IRG Singapore-MIT Alliance for Research and Technology (SMART) Center, 3 Science Drive 2, Singapore 117543

*E-mail: njcho@ntu.edu.sg

\begin{abstract}
One challenging aspect of quartz crystal microbalance (QCM) measurements is the characterization of adsorbed particles as the change in resonance frequency $(\Delta f)$ is proportional not only to the inertia of the adsorbed layer but also to that of the hydrodynamically-coupled fluid. Herein, by solving numerically the Navier-Stokes equations, we scrutinize $\Delta f$ for sparsely deposited, rigid spherical particles, that are firmly attached to an oscillating surface. The analysis is shown to be applicable to adsorbed, small unilamellar vesicles (SUVs) of controlled size under experimental conditions in which adhesion-induced vesicle deformation is negligible. The model supports a hydrodynamic explanation for the overtone dependence of $\Delta f$, and was fitted to experimental data concerning three monodisperse populations of SUVs with different average sizes ranging between 56 and $114 \mathrm{~nm}$ diameter. Using this procedure, we determined the average size of adsorbed vesicles to be within $16 \%$ of the size that was measured by dynamic light scattering experiments in bulk solution. In conclusion, this model offers a means to extract particle size from QCM-D measurement data, with applications to biological and synthetic nanoparticles.
\end{abstract}




\section{Introduction}

The quartz crystal microbalance with dissipation monitoring (QCM-D) is a measurement technique that enables highly sensitive characterization of adsorbates, and has found wide application across the interfacial sciences and biotechnology. ${ }^{1-4}$ The QCM-D operates as a label-free, acoustic sensor. Its transducing element is a thin (typically $0.3 \mathrm{~mm}$ ) quartz crystal, which is piezoelectrically driven to execute a thickness-shear oscillation at its resonance frequency (typically $5 \mathrm{MHz}$ ) and odd overtones thereof. ${ }^{5}$ In conventional measurement configurations, this resonance frequency $f$ and the width of the resonance peak $\Gamma$, or equivalently the rate of the energy dissipation $D=\pi \Gamma / f$, are measured, and changes in these measurement signals may occur due to adsorption, desorption, or structural reconfiguration events occurring on the quartz crystal surface. ${ }^{6,7}$ The oscillating quartz emits a viscously damped shear wave into the surrounding fluid, which can be either gas or liquid. The decay length of these waves (which equals $250 \mathrm{~nm}$ for a $5 \mathrm{MHz}$, AT-cut quartz crystal) is referred to as the viscous penetration depth $\delta{ }^{8}$ The penetration depth is illustrated in Fig. 2b with a contour map of the velocity field, showing non-zero velocity in a thin fluid layer attached to the wall. Owing these hydrodynamic effects, the QCM-D technique is sensitive to not only the adlayer, but also the corresponding, so-called "coupled fluid". 9 In a seminal work, Kanazawa and Gordon analyzed the effect of the coupled fluid on the QCM-D signal as a function of the viscosity and the density of the fluid. ${ }^{10}$

Extracting adlayer material and/or the geometrical properties, such as thickness, roughness or elasticity, usually requires fitting the QCM-D data with a model for the force acting on the oscillating quartz. Over the years, various models have been developed. The most widely used model is the Sauerbrey model, which is a proportionality between the resonance frequency $\Delta f$ and the adsorbed mass ${ }^{11}$ that is valid when the adlayer is perfectly flat, rigid and firmly attached to the quartz surface. While these conditions may be satisfied for small and strongly adhering molecules, it may not hold for flexible layers, composed of e.g. soft, water-rich biomacromolecules or lipid 
bilayers, where slippage occurs between the monolayers at the solid to liquid crystalline phase transition temperature. ${ }^{12}$ Modeling flexible adlayers requires including elastic and dissipative material properties. Mason et al. ${ }^{13}$ and later Voinova et al. ${ }^{14}$ extended the Sauerbrey model to include these properties, based on shear wave theory in viscoelastic media. In addition to the adlayer thickness and mass, the resulting model also provides the (frequency dependent) storage and loss moduli of the adlayer. ${ }^{14-16}$

It should be emphasized that the above mentioned models assume a laterally homogeneous film. Consequently, when the absorbed mass is laterally heterogeneous, e.g., in the form of individual particles, the validity of these models breaks down. Consequently analyzing heterogeneous layers using QCM-D requires solving the interaction between these lateral variations and the surrounding fluid using the three-dimensional hydrodynamics equations. This task has been completed for rough surfaces, ${ }^{17}$ where the hydrodynamics is analytically tractable, when the amplitude of the roughness is small compared to its wavelength. ${ }^{18}$ In addition lateral heterogeneous problems have been reduced to analytically tractable, laterally homogeneous problems using volume-averaging. This approach has been applied to porous layers, ${ }^{19}$ and to layers of tethered polymers, where in the latter case, a non-Newtonian rheological model was used to extract the polymer size and shape from the $\Delta \Gamma / \Delta f-$ ratio. ${ }^{20}$ In addition there are a few reports on numerical simulations of the hydrodynamics of adsorbed particles on oscillating surfaces, providing insight into the hydrodynamic interaction between adsorbed particles, ${ }^{21}$ and into the role of the flexibility of the bonds between the particles and the surface. ${ }^{22}$ Another approach analyzes heterogeneous layers by extrapolating QCM-D data on $(-\Delta f,-\Delta \Gamma / \Delta f)$ - coordinates to the limit of zero energy dissipation: $\Delta \Gamma=0$, which corresponds to a hypothetical layer of densely and rigidly packed particles. $^{23}{ }^{24}$ Assuming the height of this layer to be equal to the particle size, the latter is estimated using the Sauerbrey relationship. Despite a few agreements with alternative size measurements, ${ }^{35,36}$ there is no rigorous theoretical basis to relate the particle size to $-\Delta f$ in the $\Delta \Gamma=0$ limit, and it 
remains an open question as to why the extrapolation method works in certain cases, while it is unsuccessful in other cases.

Hence, there remain outstanding challenges when analyzing QCM-D data for heterogeneous layers of particles. As mentioned above, the complexity of this problem is related to the coupling between the particles and the surrounding fluid. ${ }^{17,21,25-30}$ When the particles are relatively close to each other, they interact hydrodynamically, i.e., they interact with fluid disturbances that are generated by neighboring particles. Since fluid disturbances decay over a length scale on the order of the particle radius $a$, there exists a limit of low surface coverage, where the distances between the particles are (i) sufficiently large to prevent hydrodynamic coupling between the particles and (ii) sufficiently small to ensure a measurable QCM-D signal. As compared to more dense systems, studying this limit provides advantages as it reduces the complexity of the hydrodynamics to that of a single particle, as opposed to the case where particles are closer together and hydrodynamically interact. While the dynamics of the fluid coupled to hydrodynamically interacting particles has been investigated experimentally ${ }^{41,43}$ and numerically, ${ }^{21}$ the corresponding fundamental problem of fluid coupled to hydrodynamically isolated particles has, to the best of our knowledge, not yet been addressed.

Herein, we analyze the corresponding hydrodynamics of rigid spherical particles that are firmly attached to an oscillating surface, using dimensional analysis and numerical simulation. As explained above, we circumvent the problem of hydrodynamic coupling between the particles by focusing on the limit of a very low surface coverage. Using this approach, we are able to derive a model, which allows us to extract the particle size and the particle surface coverage from QCM-D measurement data. This approach is markedly different from conventional QCM-D models, which focus on the dense limit and model the adlayer as a homogeneous film. We will show that despite the assumption of the rigidity of the particles, the model can be applied to characterize soft matter, such as lipid vesicles, under appropriate experimental conditions. 


\section{Problem Statement \& Dimensional Analysis}

We consider solid spheres (radius $a$ and mass $m_{\mathrm{P}}=\rho_{\mathrm{P}} 4 \pi a^{3} / 3$, with $\rho_{\mathrm{P}}$ the particle mass density), which are rigidly attached to the QCM-D sensor surface. Furthermore, we consider the limiting case where the spacing between the spheres is much larger than the sphere radius. In this limit, there is negligible hydrodynamic coupling between the spheres, which implies that the QCM-D force for $N$ absorbed spheres equals $N$ times the force for one isolated sphere. The QCM-D frequency shift $\Delta f$ and the bandwidth shift $\Delta \Gamma$ are related to the force $\Delta \widehat{F}$ acting on the quartz, as follows: ${ }^{31}$

$\frac{\Delta f}{f}+i \frac{\Delta \Gamma}{f}=-\frac{\Delta \widehat{F}}{i \omega \widehat{U} m_{Q}}$.

Here $\widehat{U}, f$ and $\omega=f 2 \pi$ are the velocity amplitude, the frequency and the angular frequency of the quartz, respectively, and $i=\sqrt{-1}$. The force $\Delta \widehat{F}$ in Eq. (1) is referred to as the "QCM-D force". Furthermore, for ideal resonators, $m_{\mathrm{Q}}$ is the mass of the quartz, while for real resonators $m_{\mathrm{Q}}$ is slightly different. ${ }^{31}$ The hat "^»" on $\widehat{U}$ and $\Delta \widehat{F}$ indicates a complex valued amplitude of an oscillating quantity. An oscillating (real valued) quantity $Q$ is related to its complex valued amplitude $\hat{Q}$ via $Q=\Re[\hat{Q} \exp (i \omega t)]$, where $\mathfrak{R}$ signifies the real part. As shown in Eq. (1) the real part of $\Delta \hat{F} / i \widehat{U}$ corresponds to the inertia component of the QCM-D force, which is in phase with the displacement oscillation of the quartz surface and is manifested in the $\Delta f$ response, while the imaginary part of $\Delta \widehat{F} / i \widehat{U}$ corresponds to the friction component of the QCM-D force, which is opposite to the velocity oscillation of the quartz surface and is reflected in the $\Delta \Gamma$ response. The QCM-D force $\Delta \widehat{F}=m_{P} i \omega \widehat{U}+\Delta \widehat{F}_{H}$ is composed of two parts, the inertia of the sphere (mass $m_{\mathrm{P}}$ times acceleration $i \omega \widehat{U}$ ) and the difference of the hydrodynamic force $\Delta \widehat{F}_{H}$ on the combined system of the oscillating wall and the sphere minus the hydrodynamic force on the oscillating wall without the sphere.

In supporting Section $\mathrm{S} 1$, we use dimensional analysis to show that $\Delta \widehat{F}_{\mathrm{H}}$ depends on the various variables in the following way: 
$\Delta \widehat{F}_{\mathrm{H}}=i \rho_{F} a^{3} \omega \widehat{U} \hat{\varphi}\left(\frac{\delta}{a}\right)$

Here $\delta=\left(2 v_{\mathrm{F}} / \omega\right)^{1 / 2}$ is the viscous penetration depth, $v_{\mathrm{F}}=\eta_{\mathrm{F}} / \rho_{\mathrm{F}}$ is the fluid kinematic viscosity, $\rho_{\mathrm{F}}$ is the fluid mass density, $\eta_{\mathrm{F}}$ is the fluid dynamic viscosity, and $\hat{\varphi}$ is a (yet unknown) dimensionless, complex valued function of a single dimensionless variable $\delta / a$. The imaginary unit has been added to Eq. (2) to ensure that the real and imaginary parts of $\hat{\varphi}$ correspond to frequency shift and bandwidth shift, respectively. Eq. (2) is derived under the assumption of a small Reynolds number $\operatorname{Re}=|\widehat{U}| a / v_{F}$. This condition is satisfied under typical liposome adsorption conditions; see Supporting Section S1. A small Reynolds number renders the hydrodynamic equations linear [Eq. (3)], such that hydrodynamic force $\Delta \widehat{F}_{\mathrm{H}}$ is a linear superposition of the contributions from the individual overtones, i.e., the overtones are decoupled. It is furthermore emphasized that in the linear, small amplitude regime, the QCM-D force amplitude $\Delta \widehat{F}$ is proportional to the velocity amplitude $\widehat{U}$, and the latter does affect the sensor response, which measures the ratio between $\Delta \widehat{F}$ and $\widehat{U}$; see Eq. (1).

\section{Hydrodynamic Simulations}

We simulate the three-dimensional (3D) and time-dependent hydrodynamics around a solid sphere (radius $a$ ), which is rigidly attached to an oscillating QCM-D surface. We refer to the combined system of the sphere and the QCM-D surface as "the substrate". The substrate oscillates with a velocity $|\widehat{U}| \cos (\omega t)$. As presented in Fig. 1a, the computational domain consists of a rectangular box, which is attached to the QCM-D sensor surface. The sizes of the computational domain in the horizontal directions are both equal to $L$, while the domain size in the vertical direction equals $L_{z}$. The coordinate system is also shown in Fig. 1a. The $x$ direction coincides with the direction of the oscillation, which is parallel to the wall. The $y$ direction is parallel to the wall and normal to the $x$ direction. The $z$ direction is normal to the wall.

As argued above, we assume a sufficiently small Reynolds number Re $=|\widehat{U}| a / v_{F}$, such that 
the nonlinear, advection term in the Navier-Stokes equation of fluid motion is negligible, which then reduces to the linear Stokes equation:

$$
\frac{\partial \boldsymbol{u}}{\partial t}=-\frac{1}{\rho_{F}} \boldsymbol{\nabla} p+v_{F} \nabla^{2} \boldsymbol{u}+\omega|\widehat{U}| \sin (\omega t) \boldsymbol{\delta}_{\mathrm{x}}
$$

Here $\boldsymbol{u}$ is the fluid velocity vector, which has components $\left(u_{\mathrm{x}}, u_{\mathrm{y}}, u_{\mathrm{z}}\right), p$ is the fluid pressure and $\boldsymbol{\delta}_{\mathrm{x}}$ is the unit vector in the $x$ direction. Eq. (3) described the flow in the reference frame, that is attached to the oscillating substrate. In this frame, the substrate appears steady, which offers advantages when solving the hydrodynamics as compared to the frame in which the substrate is moving. In the co-moving reference frame the Stokes equation [Eq. (3)] contains one additional body force term, which is proportional to minus the acceleration of the substrate: $\omega|\widehat{U}| \sin (\omega t) \boldsymbol{\delta}_{\mathrm{x}}$.

The solution to Eq. (3) is numerically approximated, by employing a Lattice Boltzmann (LB) scheme. Details of this scheme are given in supporting Section S2. The 3D space and time are discretized on a four dimensional hyper-cubic grid, with a spatial and temporal lattice spacing of $\Delta x$ and $\Delta t$, respectively. In this grid, the sphere is represented as a $3 \mathrm{D}$ staircase, which is illustrated in Fig. 1b. The flow is initialized using the solution for the oscillating flat plate, without the sphere $\boldsymbol{u}(t=0)=|\widehat{U}| \cos (z / \delta) \exp (-z / \delta) \boldsymbol{\delta}_{\mathbf{x}} \cdot{ }^{32}$ After startup, the simulated flow undergoes a transition period before reaching a periodic, oscillatory state. The transition period $\sim a^{2} / v_{\mathrm{F}}$ is small compared to one oscillation period $T$. Simulating two oscillation periods is therefore sufficient to ensure that the system has reached the periodic, oscillatory state.

From the simulated, time-dependent (real valued) hydrodynamic force $F_{\mathrm{H}}(\mathrm{t})$ that acts on the substrate, we compute the complex valued QCM-D force amplitude $\Delta \hat{F}$ by Fourier transformation. Details of this procedure are outlined in supporting Section S3. As defined in Eq. (1), the real part of $\Delta \widehat{F} / i \widehat{U}$ corresponds to the inertia force of the particle and that of the coupled fluid, that is responsible for $\Delta f$, while the imaginary part of $\Delta \hat{F} / i \widehat{U}$ corresponds to the hydrodynamic friction, that is responsible for $\Delta \Gamma$.

As we are interested in $\Delta f$ as a function of the scaled penetration depth $\delta / a$, we vary the latter 
parameter between 0.6 and 4.6 , by varying the particle radius $a / \Delta x$ between 10 and 20 and by varying the frequency $f \Delta t$ between $0.25 \times 10^{-4}$ and $4 \times 10^{-4}$, while keeping the viscosity fixed at $v_{\mathrm{F}}=$ 1/6 $\Delta x^{2} / \Delta t$. The fluid mass density $\rho_{\mathrm{F}}$ has the value of one in the simulations. An overview of the parameters is given in supporting Table S1. The absolute value of the velocity amplitude is $|\widehat{U}|=10^{-}$

${ }^{4} \Delta x / \Delta t$, which ensures a small Reynolds number, i.e., $\operatorname{Re}=|\widehat{U}| a / v_{F} \leq 1.2 \times 10^{-2}$, which validates the use of Eq. (3). To verify that the staircase approximation (Fig. 1b) is sufficiently accurate, i.e., that we are using sufficient grid resolution, and that the domain is sufficiently large, we validate in supporting Section S4 the accuracy of the present numerical method, by comparing simulations to analytical solutions for two test cases (see supporting Tables S2 and S3 and supporting Fig. S2). In addition, we compare simulations of the sphere on the oscillating wall by using different grid resolutions (see Table S1 and Fig. S3), which show that for $\delta / a<3$ there is grid-independence for the frequency shift $(<1 \%)$ and a slight grid dependence for the bandwidth shift $(<10 \%)$.

\section{QCM-D Experiments}

In this work, we derive from hydrodynamics simulations a relation between the QCM-D frequency shift and the size of adsorbing spherical particles. In order to test this relation, QCM-D experiments were conducted at $25.0 \pm 0.5^{\circ} \mathrm{C}$ in order to monitor the adsorption of small unilamellar vesicles (SUVs) onto a surface, that is coated with $\mathrm{TiO}_{2}$. The coating ensures intact vesicle adsorption without rupture; see e.g. Refs. [33, 34, 35]. The QCM-D signals are recorded for overtones $n=3,5$, 7, 9, and 11. The first overtone is omitted due to the anomalous radial sensitivity of this particular sensor for $n=1$, as compared to the other overtones. ${ }^{36}$

Details of the materials and methods are provided in supporting Section S6. Briefly, SUVs are produced by mixing 1,2-dioleoyl-sn-glycero-3-phosphocholine lipids with an aqueous, buffer solution ( $\mathrm{pH} 7.5$ ), containing $150 \mathrm{mM} \mathrm{NaCl}$, and extruding the mixture through a membrane with either $30 \mathrm{~nm}, 50 \mathrm{~nm}$ or $100 \mathrm{~nm}$ pores. We refer to these vesicles as SUV1, SUV2 and SUV3, and 
properties are summarized in Table 1. The resulting nominal (intensity-weighted) vesicle radii are measured with dynamic light scattering (DLS) to be $27 \mathrm{~nm}, 38 \mathrm{~nm}$ and $57 \mathrm{~nm}$, respectively. The corresponding size distributions are presented in supporting Fig. S4.

It is known that lipid vesicles deform into a dome shape upon adsorption, due to the van der Waals surface adhesion force. ${ }^{37}$ It is also known that in the present system, the surface adhesion force increases with the ionic strength, due to the screening of repulsive electrostatic forces, since both $\mathrm{TiO}_{2}$ and DOPC are negatively charged in aqueous, buffer solution; see, e.g., Ref. [38]. Since our focus is on spherical particles, we mix the vesicles in a buffer solution, with a relatively low ionic strength: $75 \mathrm{mM}$, which reduces the adhesion force, and induces a negative osmotic pressure, that counteracts and minimizes possible shape deformations ${ }^{39}$. A previous study, based on QCM-D and localized surface plasmon resonance experiements, showed that under similar ionic strength/osmotic pressure conditions, SUVs adhere firmly to the $\mathrm{TiO}_{2}$ surface and remain nearly spherical. $^{35}$

It is noted that trans-membrane ion diffusion, through the DOPC membrane may result in equilibration of the internal and the external ion density. However, the equilibration time scale, which is limited by the trans-membrane diffusivity of sodium (see Ref. [40] and references therein), which is orders of magnitude smaller than that for chloride,,$^{41}$ is estimated in the range $10^{3}$ $10^{7} \mathrm{~s}$, depending on the measurement technique and lipid used. Since this time scale is, on average, beyond the current experimental measurement period, we expect a trans-membrane osmotic pressure difference during the experiment.

In order to elucidate the effect of ionic strength on vesicle shape, we conducted a control QCM-D vesicle adsorption experiment, where SUV2 were mixed in aqueous, buffer solution with a relatively high ionic strength: $250 \mathrm{mM} \mathrm{NaCl}$.

To verify that in low ionic strength $(75 \mathrm{mM})$ the liposomes (i) are firmly adhering to the substrate (ii) are assuming a spherical shape and (iii) are not deformed by hydrodynamic forces, we 
estimate the various forces that act on the vesicles, which are the surface adhesion force: $F_{\mathrm{A}}=E_{\mathrm{A}} a$ $=4 \times 10^{-12} \mathrm{~N}$, the membrane bending force: $F_{\mathrm{B}}=8 \pi \kappa / a=6 \times 10^{-11} \mathrm{~N},{ }^{37}$ the osmotic pressure force: $F_{\mathrm{O}}=4 \pi a^{2} \Delta c R T=7 \times 10^{-9} \mathrm{~N}^{37}$ and the hydrodynamic force: $F_{H}=\left|\Delta \hat{F}_{\mathrm{H}}\right|=\rho_{F} a^{3} \omega|\widehat{U}|\left|\hat{\varphi}\left(\frac{\delta}{a}\right)\right|=$ $1 \times 10^{-11} \mathrm{~N}$. Here $E_{\mathrm{A}}=6 \times 10^{-5} \mathrm{Jm}^{-2}$ is the adhesion energy density between lipid bilayer and $\mathrm{TiO}_{2}$ surface (estimated in Ref. [38]), $a=6 \times 10^{-8} \mathrm{~m}$ is the vesicle radius (for SUV3 see Table 1), $\kappa=10^{-19}$ $\mathrm{J}$ is the bending energy, $\Delta c=150 \mathrm{~mol} \mathrm{~m}^{-3}$ is the ion concentration difference over the bilayer, $R=$ $8.3 \mathrm{~J} \mathrm{~K}^{-1} \mathrm{~mol}^{-1}$ is the ideal gas constant, $T=295 \mathrm{~K}$ is the temperature, $\rho_{\mathrm{F}}=10^{3} \mathrm{~kg} \mathrm{~m}^{-3}$ is the fluid mass density, $\omega=2 \pi f_{0}$ is the angular frequency of the quartz, $f_{0}=5 \times 10^{6} \mathrm{~s}^{-1}$ is the fundamental frequency of the quartz, $|\widehat{U}|=\omega d=6 \times 10^{-2} \mathrm{~m} \mathrm{~s}^{-1}$ is the velocity amplitude of the quartz, $d=2 \times 10^{-9}$ $\mathrm{m}$ is the displacement amplitude of the quartz, ${ }^{42}$ and $\left|\hat{\varphi}\left(\frac{\delta}{a}\right)\right|=30$ is estimated from Fig. 3a below.

Since the osmotic force is several orders of magnitude larger than the adhesion force: $F_{\mathrm{O}}>>$ $F_{\mathrm{A}}$ and the hydrodynamic force: $F_{\mathrm{O}}>>F_{\mathrm{H}}$, the vesicles are likely to assume a (nearly) spherical shape, and do not experience shape fluctuations. In addition, the time scale associated with transmembrane water diffusion, based on permeability measurements, is in the range of $10^{-4}-10^{-3} \mathrm{~s},{ }^{43}$ which is several orders of magnitude larger than one oscillation period $2 \times 10^{-7} \mathrm{~s}$, prohibiting volume changes, that are associated with shape fluctuations.

The adhesion force (estimated at $150 \mathrm{mM}$ ionic strength in Ref. [38]) is of the same order of magnitude as the hydrodynamic force $F_{\mathrm{H}}$, which means that vesicles may be subject to sliding or rocking motion. Below we discuss the magnitude of these effects, based on a comparison between experimental and numerical results.

\section{$\underline{\text { Results and Discussion }}$}

\section{$\underline{\text { Simulations }}$}

In Fig. 2a, we visualize the $x$-component of the simulated fluid velocity $u_{\mathrm{x}}$ in the vertical $x z$-plane, 
intersecting the sphere on the oscillating wall. The snapshot is taken at time $t=7 T / 4$, where $T=1 / f$ is one oscillation period. This is the moment, when the quartz velocity equals zero. The scaled penetration depth in this case is $\delta / a=0.6$. For reference we plot in Fig. $2 b$ the theoretical velocity field next to an oscillating wall without sphere, using the same parameters: $u_{\mathrm{x}}(t=7 T / 4)=$ $|\widehat{U}| \sin (z / \delta) \exp (-z / \delta)$. In Fig. 2c we plot $u_{\mathrm{x}}$ profiles on vertical line traces. The profile directly above the sphere [marked by (1) in Fig. 2a] is somewhat different from the profile directly above the plane [away from the sphere; marked by (2) in Fig. 2a] with a stronger reversed flow region, which corresponds to a vortex that is being shed from the sphere each cycle; see supporting Movie S1. For comparison we also plot the theoretical profile in Fig. 2c [marked by (3) in Fig. 2b], which matches the numerical solution away from the sphere very well.

Fig. 3 shows the complex valued, scaled QCM-D force $\Delta \widehat{F} /\left(i \rho_{F} a^{3} \omega \widehat{U}\right)$. As defined in Eq. (1) the real and imaginary parts of the scaled QCM-D force correspond to the QCM-D frequency and bandwidth shifts. Fig. 3a shows the real part $\mathfrak{N}$ as a function of $\delta / a$. It is seen that, for $\delta / a<3$, the data are well described by the following linear function of $\delta / a$ :

$\mathfrak{N}\left(\frac{\Delta \hat{F}}{i \rho_{F} a^{3} \omega \widehat{U}}\right)=\left[4.2 \frac{\rho_{P}}{\rho_{F}}+4.8+6.7 \frac{\delta}{\mathrm{a}}\right]$.

The first term within the square brackets corresponds to an inertia force that is proportional to the particle mass (Sauerbrey term), and the last two terms correspond to two force contributions from the coupled fluid. The first is an inviscid contribution, which scales with the particle volume $\sim a^{3}$, and the second is a viscous contribution, which scales with the particle area times the penetration depth $\sim a^{2} \delta$. Inserting Eq. (4) into (the real part of) Eq. (1) gives the following QCM-D frequency shift $\Delta f$ :

$\frac{\Delta f}{n}=-\frac{N f_{0} \rho_{F} a^{3}}{m_{Q}}\left[4.2 \frac{\rho_{P}}{\rho_{F}}+4.8+6.7 \frac{\delta}{a}\right]$

To arrive at Eq. (5) we have multiplied the single particle result [Eq. (4)] by the number of adsorbed particles $N$ and we have used that $f=f_{0} n$, where $f_{0}$ is the quartz fundamental frequency and $n$ is the 
overtone number. For convenience, we rewrite Eq. (5) into an expression involving the particle surface fraction $\phi=\pi(a / L)^{2}$ :

$\frac{\Delta f}{n}=-\phi \frac{f_{0}}{\pi} \frac{\rho_{F}}{\rho_{Q}} \frac{a}{l_{Q}}\left[4.2 \frac{\rho_{P}}{\rho_{F}}+4.8+6.7 \frac{\delta}{a}\right]$.

Here we have used that $N=A_{\mathrm{Q}} / L^{2}$ and $m_{\mathrm{Q}}=\rho_{\mathrm{Q}} A_{\mathrm{Q}} l_{\mathrm{Q}}$, where $A_{\mathrm{Q}}$ is the quartz surface area, $L$ is the inter-particle distance, $l_{\mathrm{Q}}$ is the thickness of the quartz and $\rho_{\mathrm{Q}}$ is the mass density of the quartz.

Eq. (6) predicts that the hydration ratio $H$ of the coupled fluid mass to the total coupled mass (particle plus fluid) decreases with increasing particle size as: $H=\left(4.8+6.7 \frac{\delta}{a}\right) /\left(4.2 \frac{\rho_{P}}{\rho_{F}}+4.8+\right.$ $\left.6.7 \frac{\delta}{a}\right)$, and equals $H=0.9$ for $a=15 \mathrm{~nm}$, which is reasonably close to the measured value of $H=$ 0.8 for $a=15 \mathrm{~nm}$ liposomes. ${ }^{27}$

Note that the $\delta / a$ term within the square brackets in Eq. (6) depends on the overtone number $n$ since $\delta=\left(v_{\mathrm{F}} / \pi f_{0}\right)^{1 / 2} n^{-1 / 2}$. Thus, Eq. (6) predicts that $\Delta f / n$ depends on overtone, which is typically observed for heterogeneous layers of adsorbed particles. ${ }^{23,24,30,44-46}$ In the literature, this overtone dependence is usually attributed to the softness of the particles, e.g., proteins, viruses and vesicles, or to the softness of the bonds between the particles and the substrate. ${ }^{22,47}$ The present result [Eq. (6)], on the other hand, supports a purely hydrodynamic explanation for the overtone dependence of $\Delta f / n$ for heterogeneous layers, which was also suggested using roughness theory ${ }^{18}$ for heterogeneous proteins films. ${ }^{46,22}$

It is emphasized that the linear relation between $\Delta f / n$ and $\delta / a$ [Eq. (6)] is valid for $\delta / a<3$, while for $\delta / a>3$, the $\Delta f / n$ is seen to deviate from linearity (Fig. 3a), which is consistent with the simulated insensitivity of $\Delta f / n$ towards $n$ for proteins with $a=6 \mathrm{~nm}$, where $\delta / a$ ranges between 42 and 12 for $n$ between 1 and $13 .^{22}$

Fig $3 b$ shows the imaginary part $\mathfrak{I}$ of the scaled QCM-D force $\mathfrak{I}\left(\Delta \widehat{F} /\left[i \rho_{F} a^{3} \omega \widehat{U}\right]\right)$ as a function of $\delta / a$. This quantity, which corresponds to the QCM-D bandwidth shift or dissipation shift, represents the dissipative force, which originates from viscous friction and is directed opposite 
to the quartz velocity. As can be seen from Fig. 3b, this friction force is a factor of four smaller than the inertia force $\mathfrak{R}\left(\Delta \widehat{F} /\left[i \rho_{F} a^{3} \omega \widehat{U}\right]\right)$ (Fig. 3a), which is consistent with previous experiments and simulation; see e.g. Ref. [24]. These data further support that softness is not a requirement for significant (overtone dependent) bandwidth shift, as is sometimes suggested in the literature; see e.g. Ref. [44]. Instead our results indicate, that these effects also occur in purely rigid systems, due to viscous forces.

\section{Vesicle Adsorption Experiments}

QCM-D experiments are conducted using small unilamellar vesicles (SUVs). The goal of the experiments is to demonstrate the utility of Eq. (6) to extract the size of spherical particles from QCM-D measurement data. We use SUVs with nominal (intensity weighted) radii of $27 \mathrm{~nm}$ (SUV1), $38 \mathrm{~nm}$ (SUV2) and $57 \mathrm{~nm}$ (SUV3); see Table 1. All vesicles are prepared in buffer solution with $150 \mathrm{mM}$ ionic strength. Prior to the QCM-D experiment the vesicles are mixed in buffer solution with an ionic strength of $75 \mathrm{mM}$ (low ionic strength). We conducted one additional experiment where SUV2 was mixed in buffer solution with a high ionic strength of $250 \mathrm{mM}$.

We start by analyzing the QCM-D data for SUV2 in low ionic strength. Figs. 4a and 4b show the measured frequency shift $-\Delta f / n$ and bandwidth shift $\Delta \Gamma / n$ as functions of time $t$ for various overtones $n$. Note that in this work we plot the negative frequency shift $-\Delta f / n$, since this quantity is itself positive. Also, while the QCM-D instrument measures the dissipation shift $\Delta D$, in this work we report the bandwidth shift $\Delta \Gamma=n f_{0} \Delta D / \pi$, as this quantity is dimensionally equivalent to the frequency shift. The vesicles are added at $t=0 \mathrm{~min}$, and as expected, monotonic adsorption is observed, reflected by the monotonically increasing $-\Delta f / n$, that saturates roughly 20 min after starting vesicle injection. In agreement with our numerical results (Fig. 3) and with previous experimental results; see e.g. Ref. [24], the measured bandwidth shift (Fig. 4b) is a few-fold smaller than the measured frequency shift (Fig. 4a). Furthermore $\Delta \Gamma / n$ shows slight, non-monotonic 
behavior, which has previously been observed for particulate adsorbates. ${ }^{24}$

In order to apply Eq. (6) to extract the size of spherical particles from QCM-D data, we focus on the initial adsorption phase, during which there is low particle surface coverage. Fig. 4c shows that, for $t<0.8 \mathrm{~min}$, the $-\Delta f / n$ at the odd overtones are linear functions of time. Assuming a constant adsorption rate, the linearity of $-\Delta f / n(t)$ implies that there is negligible hydrodynamic coupling between the adsorbed particles, i.e., the hydrodynamic effect of an individual particle on the QCM-D does not depend on the number of adsorbed particles. Fig. 4c shows that $-\Delta f / n(t)$ is linear up to $\sim 25 \mathrm{~Hz}$ and by inserting into Eq. (6): $-\Delta f / n=25 \mathrm{~Hz}, l_{\mathrm{Q}}=3 \times 10^{-4} \mathrm{~m}, \rho_{\mathrm{Q}}=2.65 \times 10^{3} \mathrm{~kg} \mathrm{~m}^{-}$ ${ }^{3}, \delta=1.5 \times 10^{-7} \mathrm{~m}, a=4 \times 10^{-8} \mathrm{~m}, n=3, f_{0}=5 \times 10^{6} \mathrm{~Hz}$ and $\rho_{\mathrm{P}}=\rho_{\mathrm{F}}=10^{3} \mathrm{~kg} \mathrm{~m}^{-3}$ we find the corresponding particle surface fraction of $\phi=0.01(1 \%)$, which is equivalent to an average, interparticle distance $L$ of 19 particle radii $a$. This inter-particle distance supports a negligible hydrodynamic interaction. It is noted that the final frequency shift at saturation: $-\Delta f / n=125 \mathrm{~Hz}$ for $n=3$ (Fig. 4a), is smaller than what Eq. (6) would predict. This discrepancy reflects that the hydrodynamic force per particle diminishes, as the particles get closer together, due to overlap of the fluid that is coupled to neighboring particles. In the limit when particles are closely together, the second and the third terms within the square brackets of Eq. (6) diminish:

$\frac{\Delta f}{n}=-4.2 \phi \frac{f_{0}}{\pi} \frac{\rho_{P}}{\rho_{Q}} \frac{a}{l_{Q}}$

For the saturation frequency of $-\Delta f / n=125 \mathrm{~Hz}(n=3$ in Fig. 4a), Eq. (7) predicts a surface coverage of $\phi=0.37$ (37\%). However inserting $\phi=0.37$ into our low-coverage relation [Eq. (6)] gives $-\Delta f / n$ $=1011 \mathrm{~Hz}$, which is an order of magnitude larger than the measured value of $125 \mathrm{~Hz}$. This example illustrates that Eq. (6) can only be applied at low particle surface coverage. It is noted that a 10 -fold discrepancy has previously been observed between the mass equivalent of the QCM-D frequency shift and alternative measurements of the adsorbed liposome mass, based on for instance the surface plasmon resonance technique; see e.g. Ref. [48].

In Fig. $4 \mathrm{~d},-\Delta f / n$ is plotted as a function of the penetration depth $\delta=\left(v_{\mathrm{F}} / \pi f_{0}\right)^{1 / 2} n^{-1 / 2}$ at a fixed 
time of $t=0.4 \mathrm{~min}$. Since, in the linear adsorption regime, $-\Delta f / n(\delta)$ is independent of time (up to a proportionality constant), the exact value of $t$ has no effect on the following analysis. For $\delta / a<3$ the modeled frequency shift $-\Delta f / n$ [Fig. $3 \mathrm{a}$ and Eq. (6)] contains two terms, i.e., a constant term and a linear term (as functions of $\delta$ ), while for $\delta / a>3$ the modeled frequency shift deviates from linearity. Consequently, we can obtain two parameters (particle radius $a$ and particle surface fraction $\phi$ ) by fitting Eq. (6) to the experimental data in Fig. 4d, excluding $n=3$, which corresponds to $\delta / a>3$, as seen in Fig. 6a below. We thereby obtain $\phi=5.6 \times 10^{-3}(0.56 \%)$ and $a=32 \mathrm{~nm}$, which is within $16 \%$ of the value $a=38 \mathrm{~nm}$, measured by DLS (see supporting Fig. S4). The small discrepancy may partly be attributed to the fact, that smaller vesicles diffuse faster towards the surface than larger vesicles, which would result in a slightly smaller mean vesicle radius on the surface (measured by QCM-D) than in the bulk solution (measured by DLS). ${ }^{49}$

Next we compare Eq. (6) to an alternative method to extract particle size from QCM-D data. ${ }^{23}$ This method, which is illustrated in Fig. 5, involves extrapolating the QCM-D data on the (- $\Delta f / n,-$ $\Delta \Gamma / \Delta f)$ - plane to the limit of zero energy dissipation: $\Delta \Gamma=0$. The intercept $-\Delta f^{*} / n$ of the extrapolation with the horizontal axis is interpreted as that of a rigid film with a thickness of twice the particle radius. The radius can thus be found by invoking the Sauerbrey relation:

$\frac{\Delta f^{*}}{n}=-\frac{\rho_{P}}{\rho_{Q}} \frac{2 a}{l_{Q}}$.

For SUV2 in low ionic strength (Fig. 5), we find $a=20 \mathrm{~nm}$, which is $50 \%$ smaller than the DLS result: $a=38 \mathrm{~nm}$. This example illustrates that, at high particle surface coverage, QCM-D data interpretation is complicated by hydrodynamic interactions between the particles. Therefore, the effective film height [assumed $2 a$ in Eq. (8)] may be sensitive to the configuration of the particles on the QCM-D surface.

We continue to experimentally verify our model [Eq. (6)] using two additional vesicles: SUV1 and SUV3 (see Table 1), with nominal (intensity weighted) radii of $a=28$ and $57 \mathrm{~nm}$, respectively, measured by DLS (see supporting Fig. S4). Experimental $\Delta f$ data are plotted in Fig. 6a, together 
with the numerical data. Here the experimental frequency shift as well as the penetration depth are scaled with the vesicle radius, obtained from DLS, and the unknown particle surface fraction $\phi$ is found by fitting Eq. (6) to the experimental data. The experimental $\Delta f$ data agree well with Eq. (6), provided that $\delta / a<3$.

To extract the particle size we fit Eq. (6) to the $(\delta,-\Delta f / n)$ data. Following this procedure we find for SUV1, SUV2 and SUV3 a particle radius of $a=24 \mathrm{~nm}, 32 \mathrm{~nm}$ and $53 \mathrm{~nm}$, which are within $16 \%$ of $a=28 \mathrm{~nm}, 38 \mathrm{~nm}$ and $57 \mathrm{~nm}$, respectively, obtained from DLS. These results confirm the applicability of Eq. (6) to determine particle radius $a$ from QCM-D data. This furthermore supports that the assumptions underlying Eq. (6) are to a reasonable extent satisfied by SUV1, SUV2 and SUV3 adhering to $\mathrm{TiO}_{2}$ under low ionic strength conditions. This suggests that, in this system, the adhesion between these SUVs and the substrate is sufficiently strong to ensure rigid adhesion, but not as strong as to induce substantial deformation.

Experimental $\Delta \Gamma$ data in Fig. 6 b for SUV1, SUV2 and SUV3 are within $15 \%$ of the numerical values. The discrepancy is somewhat larger than for the frequency shift (Fig. 6a), reflecting that for these systems, the bandwidth shift is more difficult to accurately measure or simulate than the frequency shift; see also Ref. [21].

In order to scrutinize the effect of the ionic strength on the vesicle shape, we conduct a control experiment, using SUV2 ( $a=38 \mathrm{~nm}$ obtained from DLS) under high ionic strength conditions (250 $\mathrm{mM} \mathrm{NaCl}$ ). Based on a diffusion limited adsorption model: $N \sim c Q^{1 / 3} D^{2 / 3} t$ [see supporting Eq. (S3)], the number of adsorbed vesicles $N$ in high ionic strength is expected to be the same as in low ionic strength. This is because both experiments are conducted using identical SUV bulk number density $c$ and volumetric flow rate $Q$, and the (possible) change in vesicle bulk diffusivity $D$ is less than $10 \%$ under an (osmotic) constant-area deformation from spherical to ellipsoidal. ${ }^{50,51}$ In supporting Fig. S4 we use DLS to measure $D$ in different ionic strengths, which confirms insensitivity towards shape deformation. 
Fig. 6c shows the frequency shift during the initial, linear adsorption phase of SUV2 in high ionic strength. Compared to SUV2 in low ionic strength (Fig. 4c), SUV2 in high ionic strength induces a smaller $-\Delta f / n$. Assuming similar $N$ in both cases, this suggests a reduced vesicle volume, i.e., the adsorbed vesicles in high ionic strength appear to be deformed, which is consistent with increased vesicle-substrate attraction as well as osmotic pressure-induced deformation.

In order to further scrutinize the effect of high ionic strength on adsorbed vesicle shape, we analyze in Fig. 5 the corresponding QCM-D data, with the extrapolation method. ${ }^{23}$ This method involves plotting the QCM-D data on $(-\Delta f / n,-\Delta \Gamma / \Delta f)$ coordinates and intersecting the linear curve fit with the horizontal axis. The intersect may be interpreted as an effective film height, by using the Sauerbrey relation; see Eq. (8). As shown in Fig. 5, the resulting, effective film height for SUV2 in high ionic strength is $50 \%$ smaller as compared to the corresponding film height in low ionic strength, which supports that the vesicles in high ionic strength are substantially deformed as compared to the vesicles in low ionic strength.

It is noted that, since the adsorbed vesicles in high ionic strength are expected to be nonspherical, Eq. (6) cannot be applied to find the vesicle size. To illustrate this case, we plot in Figs. $6 \mathrm{a}$ and $6 \mathrm{~b}$ the experimental data for SUV2 in high ionic strength together with the corresponding data in low ionic strength. The corresponding scaled frequency shift shows a significantly smaller slope as a function of the scaled penetration depth (Fig. 6a), and the bandwidth shift is $\sim 50 \%$ smaller than for the vesicles in low ionic strength. Furthermore, when we fit Eq. (6) to the corresponding $-\Delta f / n(\delta)$ data, we find $a=190 \mathrm{~nm}$, which is five times as large as the expected value of $a=38 \mathrm{~nm}$ (DLS). This discrepancy underscores that the vesicles in high ionic strength are nonspherical and cannot be described by Eq. (6), which is valid for spherical particles only.

In summary, Eq. (6), which is based on curve fitting the outcome of simulations of the hydrodynamics around spherical particles, correctly extracts the particle size from QCM-D data for sufficiently small vesicles in low ionic strength, while for vesicles in high ionic strength the 
extracted value deviates substantially from the reference measurement, which supports substantial deformation of adsorbed vesicles in high ionic strength.

\section{Conclusion}

Based on numerical simulation and dimensional analysis, we have derived a model for the quartz crystal microbalance frequency shift $\Delta f$, due to the adsorption of spherical particles in the range: $\delta / a$ $<3$, where $a$ is the particle radius and $\delta$ is the viscous penetration depth, which corresponds to the parameter range that is accessible for adsorbed particles, such as globular proteins, viruses, colloids and vesicles. The model considers the limit of a low surface coverage, i.e, a large inter-particle distance, such that hydrodynamic coupling and mechanical interactions between particles may be ignored. The model explains the experimentally observed overtone $n$ dependence of $\Delta f / n$ due to a dependence of the coupled fluid on $\delta$. The model can be fitted to QCM-D data in order to extract the size of spherical particles and the particle surface coverage. Using this procedure, we determined the radius of three differently sized vesicles within $16 \%$ of DLS measurements, which supports that these vesicles behave hydrodynamically similar as rigid spheres. This was accomplished by minimizing the effects of adhesion-induced vesicle deformation, through a tuning of the ionic strength. In conclusion, the present model allows extracting heterogeneous adlayer properties from QCM-D data, with applications across a wide range of biological particulates (e.g., exosomes, viruses) and synthetic nanoparticles. Furthermore the present numerical simulation methodology can be applied to alternative adlayer configurations, supporting the design of for instance bio-inspired macromolecular materials. ${ }^{52}$

\section{$\underline{\text { Acknowledgement }}$}

This article is dedicated to the memory of Dr. Kay K. Kanazawa, a long-time friend and colleague who provided stimulating advice that motivated work in this direction. We would also like to 
acknowledge funding support from the National Research Foundation (NRF2015NRF-POC001019) to N-J.C.

\section{Supporting Information}

Dimensional analysis, details of the lattice Boltzmann method, determining the QCM-D force from the simulation, accuracy of the numerical simulation, diffusion limited adsorption model, experimental materials and methods, three tables with simulation parameters and results, three figures with simulation results, one figure with dynamic light scattering results and one movie with simulation results.

\section{Author Information}

\section{Corresponding Author}

*E-mail: njcho@ntu.edu.sg.

\section{Author Contributions}

The manuscript was written through contributions of all authors. All authors have given approval to the final version of the manuscript.

\section{Notes}

The authors declare no competing financial interest.

Table 1. The vesicles, studied in this work, are extruded through filters with three different pore sizes. The nominal radius $a$ measured by dynamic light scattering is intensity weighted. The radius $a$ predicted by the model is obtained by fitting Eq. (6) to QCM-D data on $(-\Delta f / n, \delta)$-coordinates, as exemplified in Fig. 4d below. 


\begin{tabular}{|c|c|c|c|}
\hline Name & Pore Size $[\mathrm{nm}]$ & $a[\mathrm{~nm}]$ DLS & $a$ [nm] Eq. (6) \\
\hline SUV1 & 30 & 28 & 24 \\
\hline SUV2 & 50 & 38 & 32 \\
\hline SUV3 & 100 & 57 & 53 \\
\hline
\end{tabular}




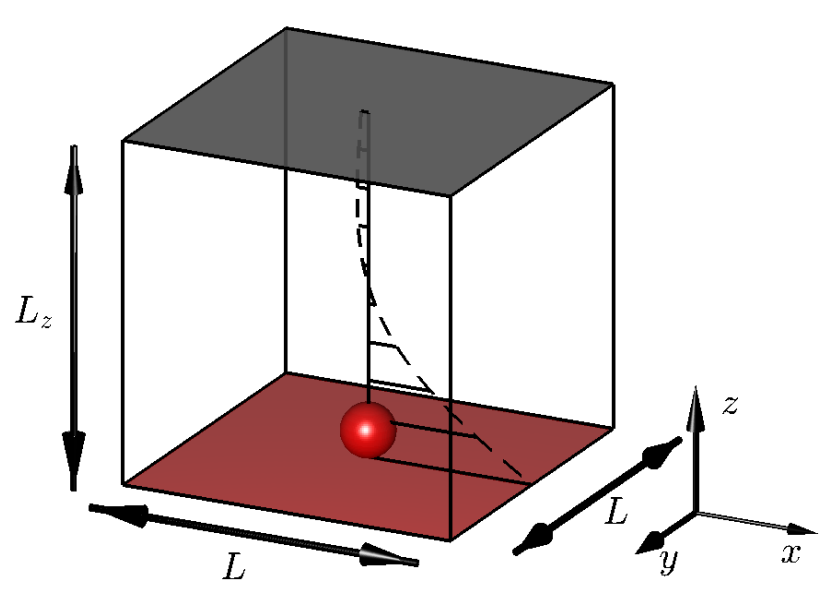

(a)

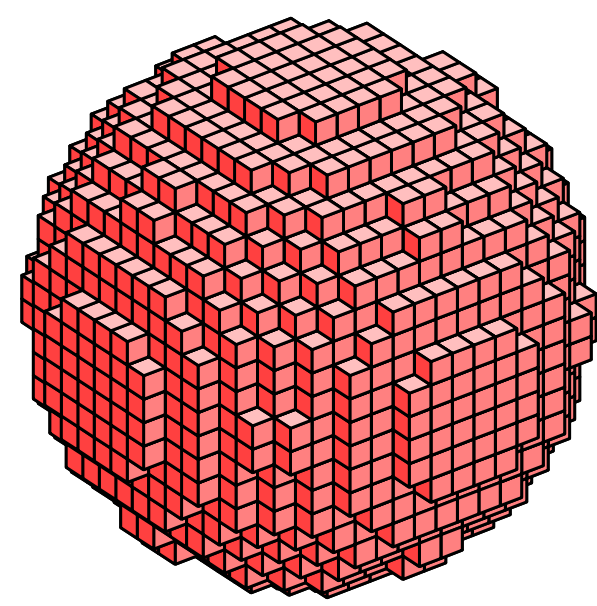

(b)

Fig. 1. Numerical setup. (a) Computational domain and coordinate system. (b) Staircase approximation of the sphere, for which the number of lattice spacings $\Delta x$ per sphere radius $a$ equals $a / \Delta x=10$. 


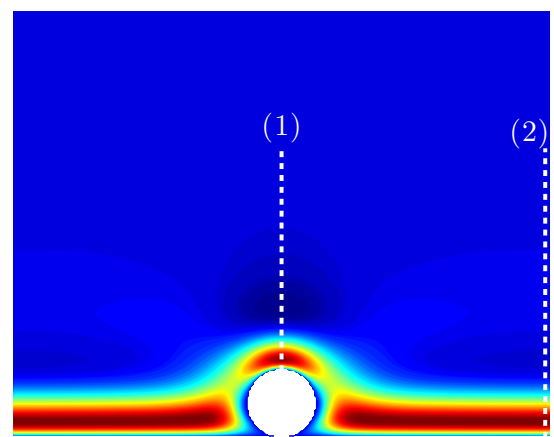

(a)

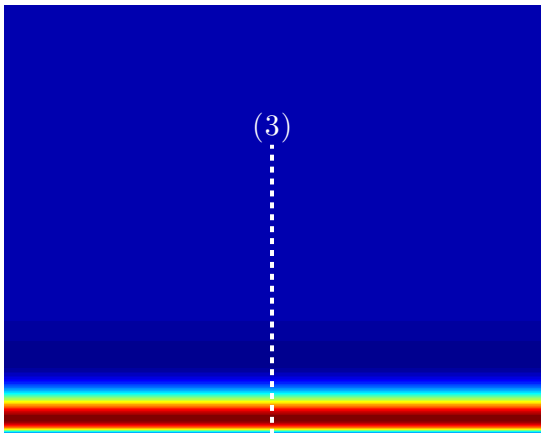

(b)

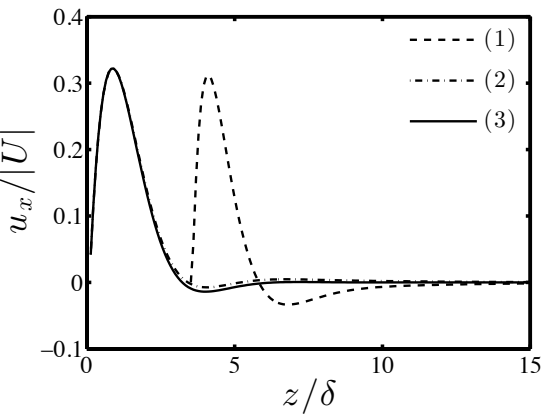

(c)

Fig. 2. Numerical simulation of a sphere attached to an oscillating wall. (a) Simulated $x$ component of the fluid velocity $u_{\mathrm{x}}$ in the $(x, z)$ plane, intersecting the sphere (see Fig. 1a). In this case, $\delta / a=0.6$ and the snapshot is taken at time $t=7 T / 4$, which is the moment when the quartz velocity equals zero. Here $\delta$ is the penetration depth, $a$ is the particle radius and $T$ is the oscillation period. (b) Theoretical fluid velocity in the absence of the sphere using the same parameters as in (a): $u_{\mathrm{x}}(t=7 T / 4)=|\widehat{U}| \sin (z / \delta) \exp (-z / \delta)$. Here $|\widehat{U}|=10^{-4} \Delta x / \Delta t$ is the absolute value of the velocity amplitude and $\Delta x$ and $\Delta t$ are the grid spacing and the time step, respectively. In panels (a) and (b), blue corresponds to $u_{\mathrm{x}}=0$ and red corresponds to $u_{\mathrm{x}}=0.3|\widehat{U}|$ (c) The $u_{\mathrm{x}}$ profiles on the line traces, which are indicated in (a) and (b) with (1), (2) and (3).

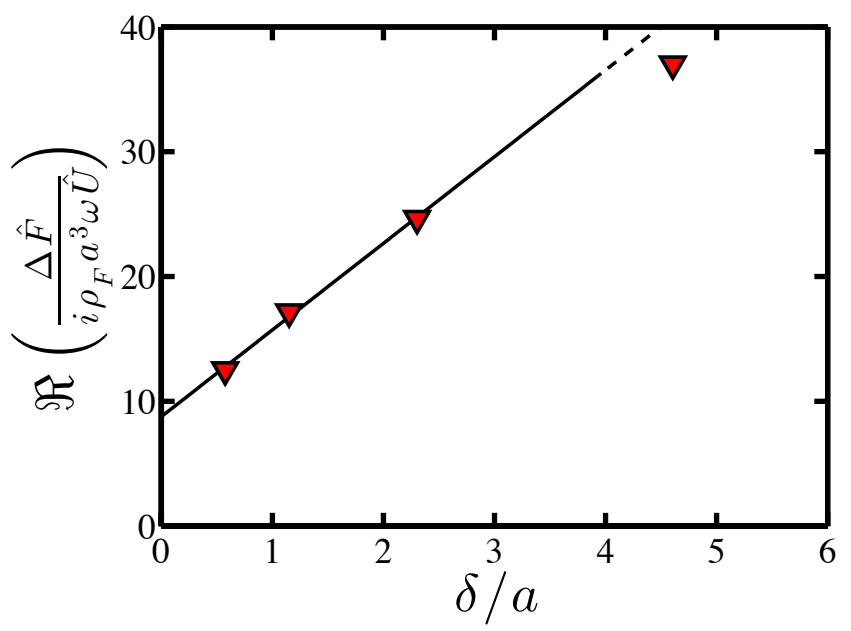

(a)

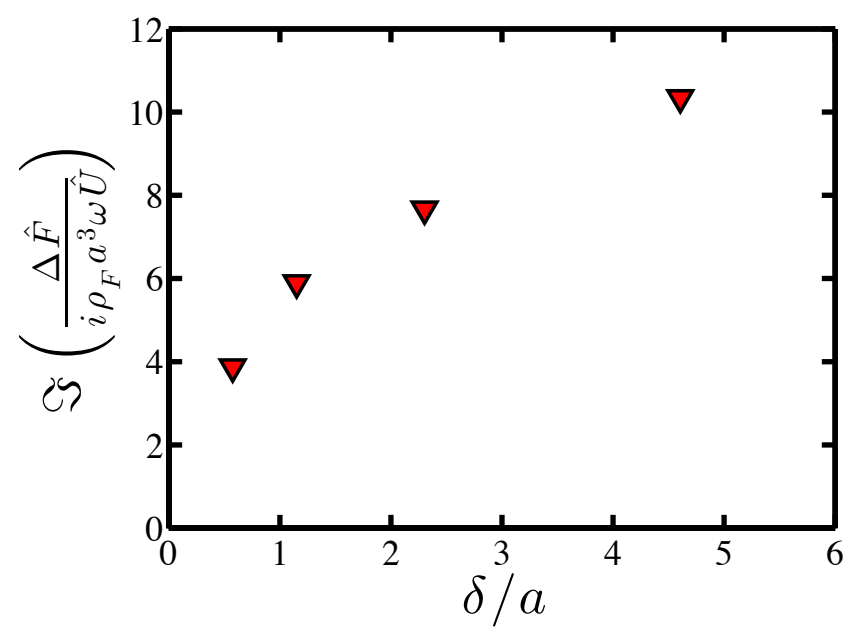

(b)

Fig. 3 Real (inertia) part (a) and imaginary (friction) part (b) of the scaled QCM-D force due to the adsorption of a sphere as a function of the scaled viscous penetration depth $\delta / a$. Here $\Delta \hat{F}$ is the complex valued QCM-D force amplitude [defined in Eq. (1)], $\rho_{\mathrm{F}}$ is the fluid mass density, $\omega$ is the angular frequency, $\widehat{U}$ is the complex valued velocity amplitude of the quartz, $a$ is the particle radius and $\delta$ is the viscous penetration depth. The markers are simulation results and the line is the model [Eq. (6)] 


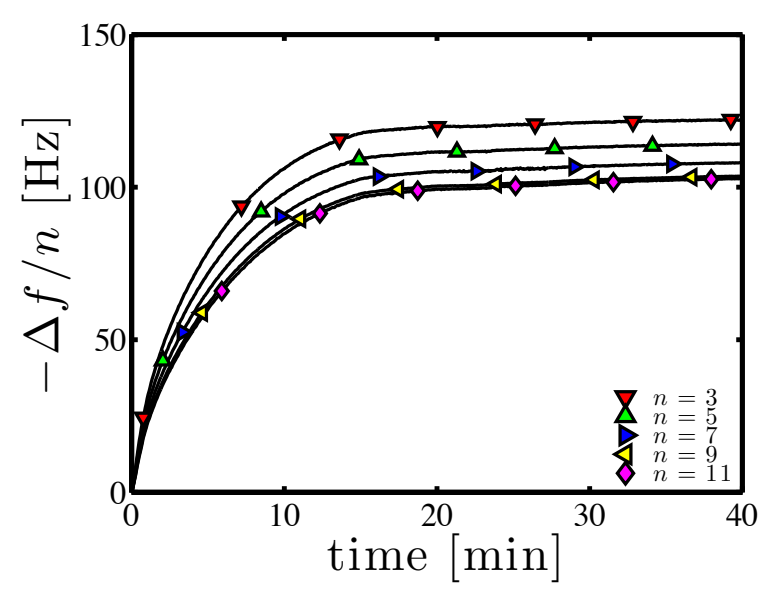

(a)

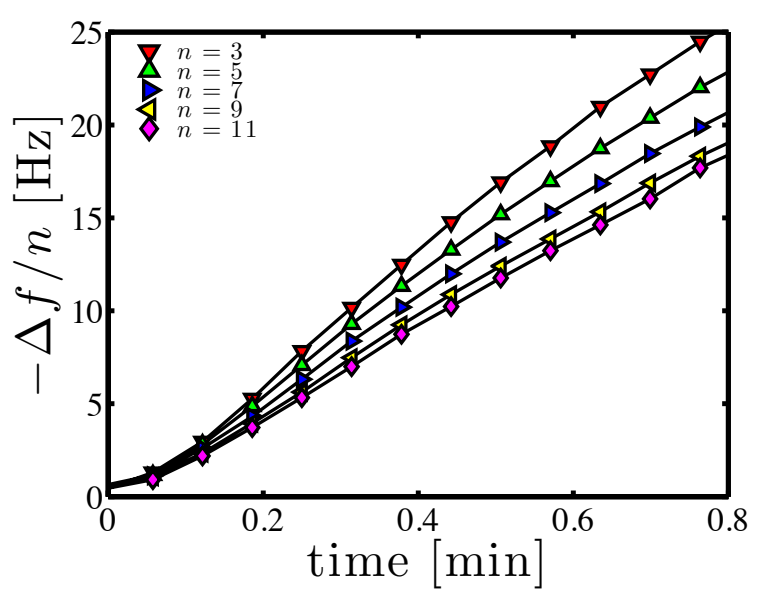

(c)

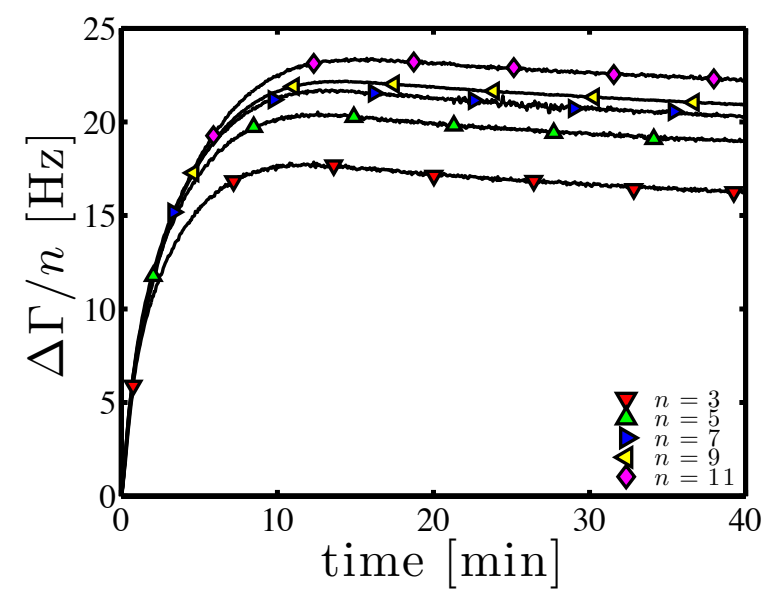

(b)

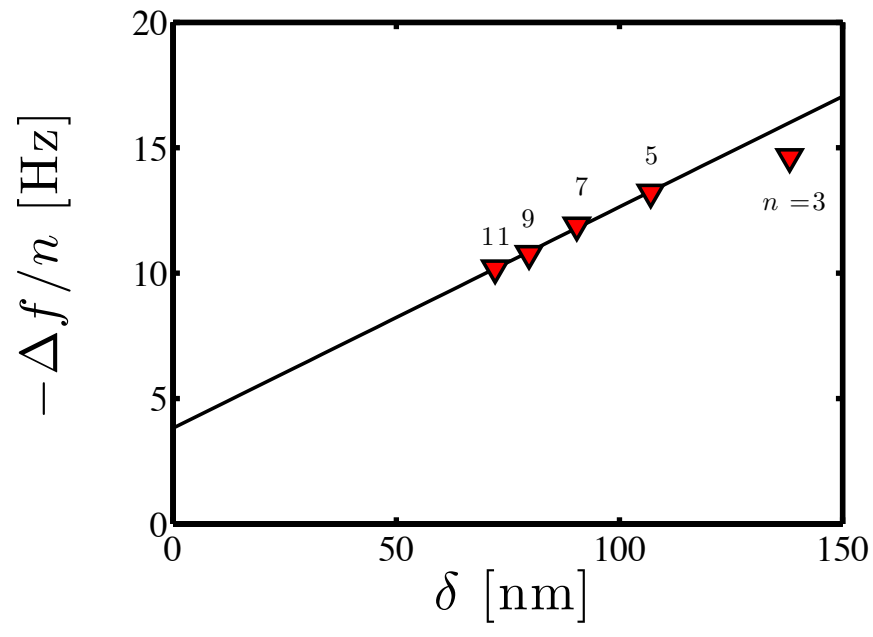

(d)

Fig. 4. Experimental QCM-D data for adsorption of SUV2 in low ionic strength. (a) Negative frequency shift (divided by overtone number $n$ ) $-\Delta f / n$ for various $n$ as a function of time. (b) Bandwidth shift (divided by overtone number $n) \Delta \Gamma / n$ for various $n$ as a function of time. (c) Negative frequency shift during the initial, linear adsorption phase. (d) $-\Delta f / n$ as a function of penetration depth $\delta=\left(v_{\mathrm{F}} / \pi n f_{0}\right)^{1 / 2}$ at $t=0.4 \mathrm{~min}$, where $v_{\mathrm{F}}$ is the fluid kinematic viscosity (of water) and $f_{0}$ is the fundamental frequency of the quartz. The solid line is a fit of Eq. (6), where the surface coverage $\phi$ and the particle radius $a$ act as fitting parameters. In all panels, the measurement noise is smaller than the marker size. 


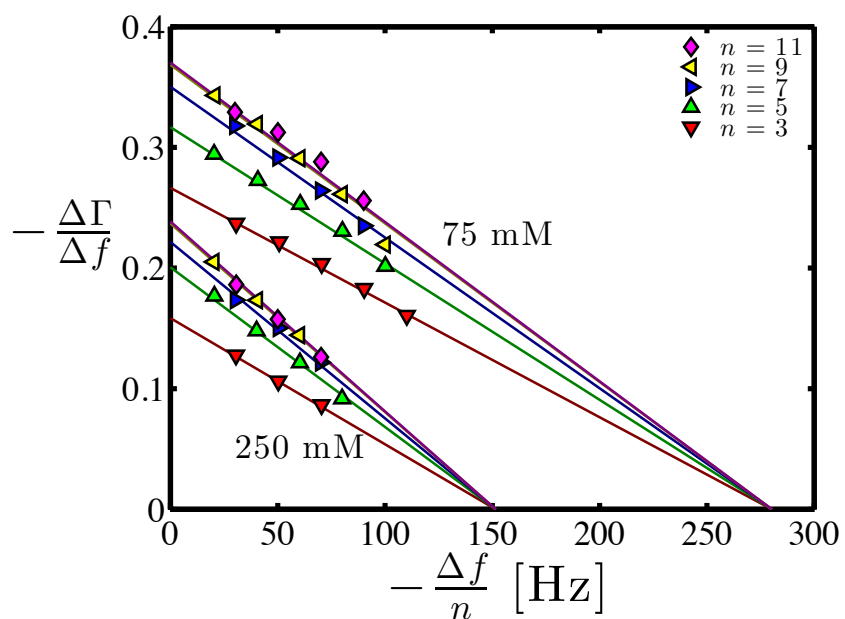

Fig. 5 Experimental QCM-D data on $(-\Delta f / n,-\Delta \Gamma / \Delta f)$ - coordinates using SUV2 in low ionic strength $(75 \mathrm{mM})$ and in high ionic strength $(250 \mathrm{mM})$. The data follow straight lines, which, when extrapolated, intercept the horizontal axis at an overtone-independent frequency shift $-\Delta f^{*} / n$, which according to Ref. [23] can be related to the particle radius: $a=-\Delta f^{*} /\left(n 2 f_{0} l_{\mathrm{Q}}\right)$, where $f_{0}$ is the quartz fundamental frequency and $l_{\mathrm{Q}}$ is the thickness of the quartz, which results in $a=20 \mathrm{~nm}$ for the vesicles in low ionic strength and $a=11 \mathrm{~nm}$ for vesicles in high ionic strength.

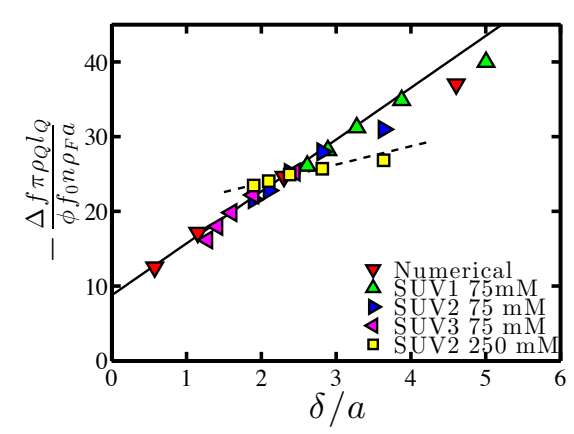

(a)

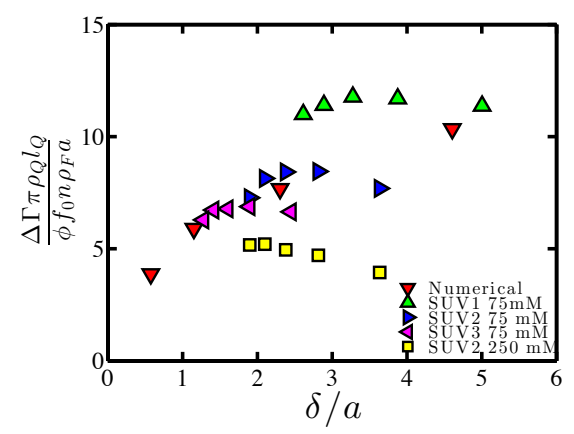

(b)

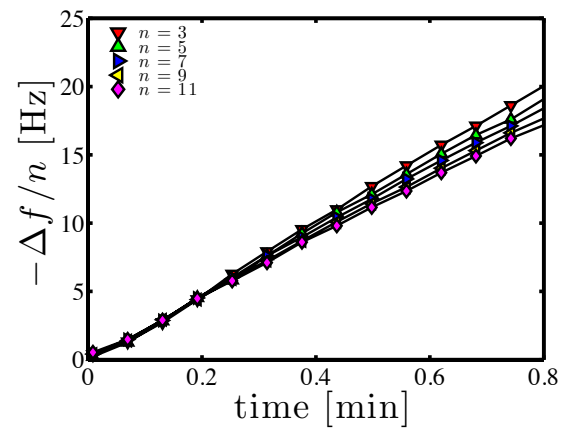

(c)

Fig. 6 Scaled frequency shift $\Delta f($ a) and scaled bandwidth shift $\Delta \Gamma$ (b) as functions of scaled penetration depth $\delta$ obtained from simulation and from QCM-D experiments, using three different vesicles. The experimental data are scaled using the particle radius $a$ from DLS (see Table 1) and using the surface coverage $\phi$, that is a fitting parameter. The overtone number is $n=3,5,7,9$ and 11 and $\rho_{\mathrm{Q}}$ is the mass density of the quartz, $\rho_{\mathrm{F}}$ is the mass density of the fluid and $l_{\mathrm{Q}}$ is the thickness of the quartz. The solid line in (a) is Eq. (6). (c) Negative frequency shift (divided by overtone number $n)-\Delta f / n$ for various $n$ as a function of time during the initial, linear adsorption phase, for SUV2 in high ionic strength. Note that the frequency shift is smaller than for SUV2 in low ionic strength (Fig. 4c), which is indicative of adhesion induced vesicle deformation. 


\section{$\underline{\text { References }}$}

1. $\quad$ S. J. Martin, V. E. Granstaff and G. C. Frye, Analytical Chemistry, 1991, 63, 2272-2281.

2. K. A. Marx, Biomacromolecules, 2003, 4, 1099-1120.

3. I. Reviakine, D. Johannsmann and R. P. Richter, Analytical Chemistry, 2011, 83, 88388848 .

4. $\quad$ N.-J. Cho, C. W. Frank, B. Kasemo and F. Höök, Nature Protocols, 2010, 5, 1096-1106.

5. A. Arnau, Piezoelectric transducers and applications, Springer, 2004.

6. M. Rodahl, F. Höök, A. Krozer, P. Brzezinski and B. Kasemo, Review of Scientific Instruments, 1995, 66, 3924-3930.

7. M. Rodahl and B. Kasemo, Review of Scientific Instruments, 1996, 67, 3238-3241.

8. M. Rodahl and B. Kasemo, Sensors and Actuators A: Physical, 1996, 54, 448-456.

9. J. Fang, C. Ren, T. Zhu, K. Wang, Z. Jiang and Y. Ma, Analyst, 2015, 140, 1323-1336.

10. K. K. Kanazawa and J. G. Gordon, Analytica Chimica Acta, 1985, 175, 99-105.

11. G. Sauerbrey, Zeitschrift Fur Physik, 1959, 155, 206-222.

12. Y. Okahata, K. Kimura and K. Ariga, Journal of the American Chemical Society, 1989, 111, 9190-9194.

13. W. P. Mason, W. O. Baker, H. J. McSkimin and J. H. Heiss, Physical Review, 1949, 75, 936.

14. M. V. Voinova, M. Rodahl, M. Jonson and B. Kasemo, Physica Scripta, 1999, 59, 391.

15. D. Johannsmann, Macromolecular Chemistry and Physics, 1999, 200, 501-516.

16. D. Johannsmann, Physical Chemistry Chemical Physics, 2008, 10, 4516-4534.

17. S. J. Martin, G. C. Frye, A. J. Ricco and S. D. Senturia, Analytical Chemistry, 1993, 65, 2910-2922.

18. L. Daikhin, E. Gileadi, G. Katz, V. Tsionsky, M. Urbakh and D. Zagidulin, Analytical Chemistry, 2002, 74, 554-561.

19. L. Daikhin and M. Urbakh, Langmuir, 1996, 12, 6354-6360.

20. A. Tsortos, G. Papadakis and E. Gizeli, Analytical Chemistry, 2016, 88, 6472-6478.

21. D. Johannsmann and G. Brenner, Analytical Chemistry, 2015, 87, 7476-7484.

22. D. Johannsmann, I. Reviakine, E. Rojas and M. Gallego, Analytical Chemistry, 2008, 80, 8891-8899.

23. E. Tellechea, D. Johannsmann, N. F. Steinmetz, R. P. Richter and I. Reviakine, Langmuir, 2009, 25, 5177-5184.

24. I. Reviakine, M. Gallego, D. Johannsmann and E. Tellechea, Journal of Chemical Physics, 2012, 136, 084702.

25. J. Vörös, Biophysical Journal, 2004, 87, 553-561.

26. L. Macakova, E. Blomberg and P. M. Claesson, Langmuir, 2007, 23, 12436-12444.

27. P. Bingen, G. Wang, N. F. Steinmetz, M. Rodahl and R. P. Richter, Analytical Chemistry, 2008, 80, 8880-8890.

28. M. Edvardsson, S. Svedhem, G. Wang, R. Richter, M. Rodahl and B. Kasemo, Analytical Chemistry, 2008, 81, 349-361.

29. I. Carton, A. R. Brisson and R. P. Richter, Analytical Chemistry, 2010, 82, 9275-9281.

30. C. Grunewald, M. Schmudde, C. N. Noufele, C. Graf and T. Risse, Analytical Chemistry, 2015, 87, 10642-10649.

31. D. Johannsmann, The Quartz Crystal Microbalance in Soft Matter Research: Fundamentals and Modeling, Springer, 2014.

32. L. D. Landau and E. M. Lifshitz, Fluid Mechanics, Pergamon Press, Oxford, UK, 1959.

33. E. Reimhult, F. Höök and B. Kasemo, Langmuir, 2003, 19, 1681-1691.

34. J. A. Jackman, G. H. Zan, Z. Zhao and N.-J. Cho, Langmuir, 2014, 30, 5368-5372.

35. J. A. Jackman, S. Yorulmaz Avsar, A. R. Ferhan, D. Li, J. H. Park, V. P. Zhdanov and N.-J. 
Cho, Analytical Chemistry, 2016, 89, 1102-1109.

36. P. J. Cumpson and M. P. Seah, Measurement Science and Technology, 1990, 1, 544.

37. U. Seifert and R. Lipowsky, Physical Review A, 1990, 42, 4768.

38. R. Tero, T. Ujihara and T. Urisu, Langmuir, 2008, 24, 11567-11576.

39. J. A. Jackman, J.-H. Choi, V. P. Zhdanov and N.-J. Cho, Langmuir, 2013, 29, 11375-11384.

40. D. Papahadjopoulos, Biochimica et Biophysica Acta (BBA)-Biomembranes, 1971, 241, 254 259.

41. S. Paula, A. G. Volkov and D. W. Deamer, Biophysical journal, 1998, 74, 319-327.

42. B. Borovsky, B. L. Mason and J. Krim, Journal of Applied Physics, 2000, 88, 4017-4021.

43. R. Fettiplace and D. A. Haydon, Physiological Reviews, 1980, 60, 510-550.

44. I. Reviakine, F. F. Rossetti, A. N. Morozov and M. Textor, Journal of Chemical Physics, 2005, 122, 204711.

45. A. L. Olsson, I. R. Quevedo, D. He, M. Basnet and N. Tufenkji, ACS Nano, 2013, 7, 78337843.

46. E. Rojas, M. Gallego and I. Reviakine, Analytical Chemistry, 2008, 80, 8982-8990.

47. D. Johannsmann, I. Reviakine and R. P. Richter, Analytical Chemistry, 2009, 81, $8167-$ 8176.

48. E. Reimhult, M. Zäch, F. Höök and B. Kasemo, Langmuir, 2006, 22, 3313-3319.

49. J. A. Jackman, G. H. Zan, V. P. Zhdanov and N.-J. Cho, The Journal of Physical Chemistry $B, 2013,117,16117-16128$.

50. $\quad$ F. Perrin, Journal De Physique Et Le Radium, 1936, 7, 1-11.

51. J. Pencer, G. F. White and F. R. Hallett, Biophysical Journal, 2001, 81, 2716-2728.

52. G. N. Pandian and H. Sugiyama, Bulletin of the Chemical Society of Japan, 2016, 89, 843868. 